\title{
The release of a distinct cell type from swarm colonies facilitates dissemination of Vibrio parahaemolyticus in the environment
}

\author{
Carolina Freitas $\mathbb{I}^{1} \cdot$ Timo Glatter $^{2} \cdot$ Simon Ringgaard $\mathbb{C}^{1}$
}

Received: 3 July 2019 / Revised: 20 August 2019 / Accepted: 25 August 2019 / Published online: 17 October 2019

(c) The Author(s), under exclusive licence to International Society for Microbial Ecology 2019

\begin{abstract}
Bacteria experience changes in their environment and have developed various strategies to respond accordingly. To accommodate environmental changes, certain bacteria differentiate between specialized cell types. Vibrio parahaemolyticus is a marine bacterium, a worldwide human pathogen and the leading agent of seafood-borne gastroenteritis. It exists as swimmer or swarmer cells, specialized for life in liquid and on solid environments, respectively. Swarmer cells are characteristically highly elongated—a morphology important for swarming behavior. When attached to surfaces it forms swarm colonies, however, it is not known how cells within swarming populations respond to changes in the external milieu and how its distinct life cycle influences its ecological dissemination. The worldwide distribution of V. parahaemolyticus accentuates the need for understanding the factors contributing to its dissemination. Here we determine the stage-wise development of swarm colonies and show how the swarm colony architecture fluctuates with changing environmental conditions. Swarm colonies act as a continuous source of cells that are released from the swarm colony into the environment. Surprisingly, the cell length distribution of released cells was very homogenous and almost no long cells were detected, indicating that swarmer cells are not released into the liquid environment but stay surface attached during flooding. Released cells comprise a distinct cell type that is morphologically optimized for swimming behavior and is capable of spreading in the liquid environment and attach to new surfaces. Release of this distinct cell type facilitates the dissemination of $V$. parahaemolyticus in the environment and likely influences the ecology of this bacterium.
\end{abstract}

\section{Introduction}

The marine environment is an important source of pathogenic bacteria worldwide. Importantly, certain bacterial pathogens can cause disease outbreaks depending on the environmental conditions. Thus, it is essential to understand what factors influence the dissemination of pathogens in the environment. An excellent example is Vibrio parahaemolyticus, which is a Gram-negative halophilic bacterium that is indigenous to

Supplementary information The online version of this article (https:// doi.org/10.1038/s41396-019-0521-x) contains supplementary material, which is available to authorized users.

Simon Ringgaard

simon.ringgaard@mpi-marburg.mpg.de

1 Department of Ecophysiology, Max Planck Institute for Terrestrial Microbiology, 35043 Marburg, Germany

2 Core Facility for Mass Spectrometry and Proteomics, Max Planck Institute for Terrestrial Microbiology, 35043 Marburg, Germany marine, estuarine, and coastal areas. It is often found in a free swimming state in liquid environments and in a sessile state when it occupies surfaces. In its sessile state it is found attached both to inert and animate surfaces such as zooplankton, fish, and shellfish $[1,2]$. The incidence of $V$. parahaemolyticus is highest in estuarine or coastal areas of the world oceans rather than in offshore sea [3-6]. Importantly, $V$. parahaemolyticus is recognized as the leading agent of seafood-borne acute gastroenteritis worldwide [2,7].

$V$. parahaemolyticus has an intricate life cycle that depends on its environmental conditions. Particularly, in liquid environments it exits as a short motile cell that is propelled by a single polar flagellum. However, when it attaches to solid surfaces, V. parahaemolyticus possesses the ability to induce a distinct differentiation program, which allows it to adapt to changes in its environment and colonize solid surfaces by means of swarm motility [1, 8-12]. One of the first steps in swarmer differentiation is inhibition of cell division, resulting in highly elongated rod-shaped filamentous swarmer cells (Fig. 1a). A second major change during swarmer differentiation is the expression of a secondary independent 
A Developmental life-cycle of Vibrio parahaemolyticus

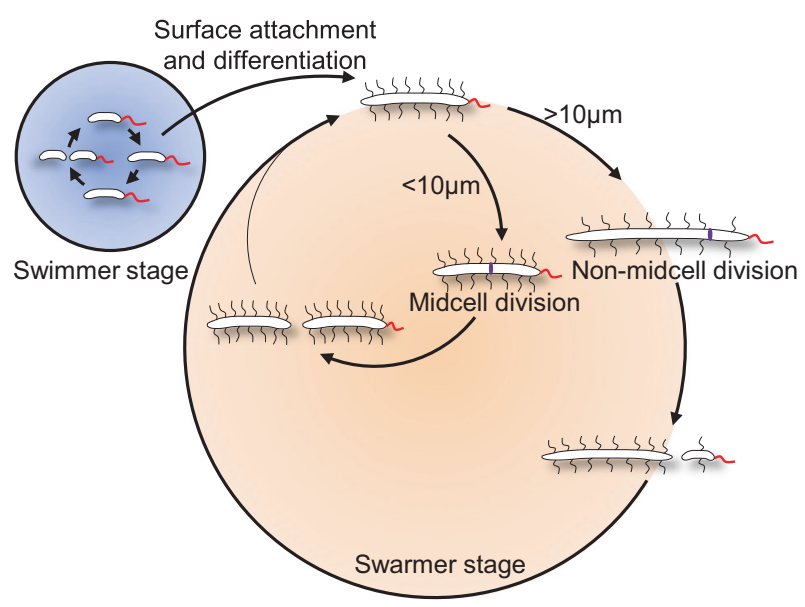

B

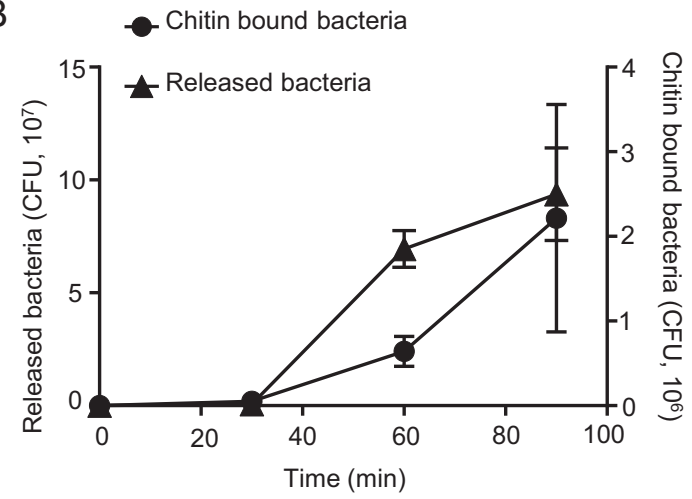

C

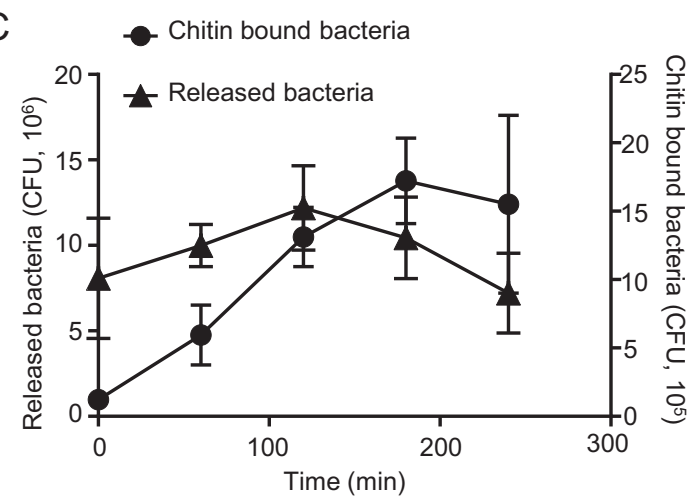

Fig. 1 Cells are released from flooded swarm colonies into their liquid surroundings. a Schematic showing the developmental life cycle of V. parahaemolyticus. Figure adapted and edited from [15]. b Graph showing the number $(\mathrm{CFU} / \mathrm{mL})$ of released cells into the liquid phase of flooded swarm colonies as a function of time (filled triangles). Graph showing number of cells (CFU/20 mg of chitin) that have attached to chitin surfaces as a function of time during flooding of swarm colonies (filled black circles). c Cells released from flooded swarm colonies were collected and their ability to attach to chitin was measured as a function of time. Graphs show the number of cells (CFU) in the liquid phase (filled triangles) and cells attached to chitin (filled circles) as a function of time. b, $\mathbf{c}$ Error bars indicate standard error of the mean (SEM) and are based on three independent biological replicates flagellum system-the laf system, which is specifically expressed in cells that have initiated the swarm program. This results in the production of a multitude of lateral flagella along the length of the cell that are important for its swarming behavior and rapid surface colonization.

Within swarm colonies there are differences in cell size according to the position of cells within a swarm colony. In the periphery of the swarm colony, cells assemble into flares that extend outward from the colony and cells are stacked in a few layers only. In the center of the swarm colony cells are stacked in multiple layers and are generally considerably shorter than cells in the flares [13-15]. Cell elongation is important for proper swarming behavior and the elongation phenotype is usually higher for species that navigate across a hard agar surface [16-20]. However, it is not clear if the difference between the cells in the center and in the periphery of a $V$. parahaemolyticus swarm colony is merely in regard to their morphology or if swarm colonies truly exists of distinct zonal regions of cells with different developmental expression profiles. Particularly, the development and differentiation of swarm colonies is unknown. Moreover, it is not known how $V$. parahaemolyticus cells within swarm colony populations respond to changes in the external milieu-particularly during fluctuations of flooding and non-flooding conditions, which is characteristic of the tidal rhythms found in estuarine areas - a common habitat for $V$. parahaemolyticus. Indeed, there have been reports suggesting that the level of $V$. parahaemolyticus in the water depends on the tide, suggesting that cells could be released from surfaces into the liquid surroundings [21, 22]. However, the release of surface attached cells into liquid environments have remained unexplored for $V$. parahaemolyticus - and for swarm colonies in general.

Furthermore, the worldwide prevalence and distribution of $V$. parahaemolyticus gastroenteritis cases emphasizes the need for understanding how this bacterium spreads in the environment and how its distinct life cycle influences its ecological dissemination. Our aim was to investigate these outstanding questions and we therefore analyzed the importance of swarm colony development and architecture on the dissemination of $V$. parahaemolyticus in the environment. We show that swarm colony development and differentiation follows a specific stage-wise progress that is divided into four stages. This ultimately results in a mature swarm colony with a highly structured architecture that consists of specific groups of cells of distinct differentiation states and properties. Importantly, we identify a distinct, highly swimming and chemotaxis proficient cell type, that is released from swarm colonies during colony flooding events. This cell type is morphologically different from elongated swarmer cells, which remain surface attached. Release of this cell type from swarm colonies facilitates the dissemination of $V$. parahaemolyticus during fluctuations in the external environment. 


\section{Materials and methods}

\section{Growth conditions and media}

In all experiments, unless otherwise stated, $V$. parahaemolyticus and $E$. coli were grown in LB medium or on LB agar plates at $37{ }^{\circ} \mathrm{C}$ containing antibiotics in the following concentrations: $100 \mu \mathrm{g} / \mathrm{mL}$ ampicillin, $5 \mu \mathrm{g} / \mathrm{mL}$ chloramphenicol for $V$. parahaemolyticus and $20 \mu \mathrm{g} / \mathrm{mL}$ chloramphenicol for E. coli.

\section{Strains and plasmids}

The strains and plasmids used in this study are listed in Tables S1 and S2, respectively. Primers used are listed in Table S3. E. coli strains DH5 $\alpha \lambda$ pir and SM10 $\lambda$ pir were used for cloning. E. coli strain SM10גpir was used to transfer DNA into $V$. parahaemolyticus by conjugation [23]. Construction of $V$. parahaemolyticus insertions were performed with standard allele exchange techniques using derivatives of plasmid pDM4 [24]. Plasmid construction is described in detail in Supplementary information.

\section{Microscopy}

For imaging of swarmer cells from the center and periphery of swarm colonies, samples were prepared essentially as described previously $[25,26]$. Particularly, for imaging of swarmer cells from the periphery, a swarming assay was performed and the edge of the swarm colony was excised from the swarm agar. This piece of swarming agar was then immediately transferred onto a microscope agarose slide with the cells facing the agarose pad. This imprints the swarming cells onto the agarose pad. After $\sim 30 \mathrm{~s}$, the agar piece was carefully removed from the agarose pad and a cover slip was mounted on top and cells immediately analyzed by microscopy. For imaging cells from the center of the swarm colony, cells were also imprinted but had to be spread over the agarose in order to get a single layer of cells before a cover slip was added. For imaging cells released from a swarm colony, $\mathrm{PBS}+1.5 \% \mathrm{NaCl}$ was gently poured into a swarming plate and after $30 \mathrm{~min}$ the liquid was collected using a pipette. Cells were harvested, resuspended in PBS, and $1 \mu \mathrm{L}$ was spotted on $1 \%$ agarose in PBS on the microscopy slide and mounted with the cover slip. In the control experiment to test for how incubation in release buffer influences the cell size distribution of cells from the center and periphery of the swarm colony, cells from center and periphery, respectively, were scrapped off with an inoculation loop and resuspended into $100 \mu \mathrm{L}$ of PBS. After 30 min incubation, $1 \mu \mathrm{L}$ was spotted on $1 \%$ agarose in PBS on the microscopy slide and mounted with the cover slip and samples were immediately analyzed by microscopy.
More details on microscopy methods can be found in the Supplementary information.

\section{Release assay and chitin attachment experiment}

In this assay, three individual swarming plates of $92 \mathrm{~mm}$ diameter were used for each time point. Swarm colonies were prepared from the same liquid culture and presented the same size. Chitin flakes of $\approx 20 \mathrm{mg}$ were added around a swarm colony and $10 \mathrm{~mL}$ PBS $1.5 \% \mathrm{NaCl}$ were poured gently into each swarm agar plate. Cells released into the PBS and cells attached to the chitin flakes were collected at 0, 30, 60 and $90 \mathrm{~min}$ after adding PBS to the swarm agar plates. To calculate the number of released cells $100 \mu \mathrm{L}$ was collected from the plate, and serial dilutions were plated for CFU (per mL) determination. To calculate the amount of cells attached to chitin, the chitin flakes were collected in a $2 \mathrm{~mL}$ tube and washed gently three times with $1 \mathrm{~mL}$ of PBS $1.5 \% \mathrm{NaCl}$. Chitin flakes were then resuspended in $100 \mu \mathrm{L}$ of PBS $1.5 \% \mathrm{NaCl}$ and serial dilutions were plated for $\mathrm{CFU}$ (per $20 \mathrm{mg}$ of chitin) determination.

\section{Flooding assay}

Twenty-five $\mathrm{mL}$ of PBS $1.5 \% \mathrm{NaCl}$ were gently poured into a $150 \mathrm{~mm}$ swarming plate and after $30 \mathrm{~min}$ the liquid was very gently collected using a pipette. After letting the plates open to dry for $4 \mathrm{~h}$ at RT, the plates were kept at nonswarming conditions-open at $24^{\circ} \mathrm{C}$. After another $18 \mathrm{hrs}$ the plates were sealed and incubated at $24^{\circ} \mathrm{C}$ for an additional $24 \mathrm{~h}$ during swarming conditions. At least three biological replicates were performed. Cells were then collected for microscopy as previously described $[25,26]$.

\section{Chemotaxis assay}

To collect cells from liquid growth, over-night cultures of $V$. parahaemolyticus wild type and $\Delta c h e W$ strains were subcultured to an initial $\mathrm{OD}_{600}$ of $\sim 0.05$ in $5 \mathrm{~mL}$ of $\mathrm{LB}$ and were grown at $37^{\circ} \mathrm{C}$ until they reached an $\mathrm{OD}_{600}$ of 1 . Released cells were collected as described above. The final $\mathrm{OD}_{600}$ was normalized between the two strains, wild type and $\Delta c h e W$, and samples were distributed in three technical replicates. Chemotaxis assays were then performed essentially as described in [27].

\section{Differential expression analyses}

To determine differentially regulated proteins in a comparison set between two conditions, a Student's $t$ test was performed (FDR $1 \%$ and $\mathrm{S} 0=0.2$ ) and a twofold regulation cut-off was further applied. The ratio of the protein intensities between two conditions was shown in $\log 2$. Clustering of individual 
protein sets was performed using five clusters and Euclidean distance. Heat maps and clustering were generated using the Perseus computational platform.

\section{Results}

\section{Cells are released from flooded swarm colonies into their liquid surroundings}

In order to understand how changing environments influence $V$. parahaemolyticus cells within swarm colonies we analyzed if cells are released from flooded swarm colonies into the environment. V. parahaemolyticus swarm colonies were very gently flooded with buffer for $90 \mathrm{~min}$ and the amount of bacteria in the liquid phase was analyzed. Importantly, we could detect high amounts of bacteria in the liquid phase $(9.4 \mathrm{E} 7 \pm 3.6 \mathrm{E} 7$ bacterial cells per $\mathrm{mL})$, suggesting that $V$. parahaemolyticus cells are released from swarm colonies into the liquid surroundings. To make sure that cells present in the liquid phase did not only originate from the initial flooding of the swarm colony, we performed a time-course experiment, where we measured the amount bacteria present in the liquid phase as a function of time (Fig. 1b). Indeed, there was a time-dependent accumulation of cells in the liquid surroundings (Fig. 1b), showing that $V$. parahaemolyticus cells are released from flooded swarm colonies into their liquid surroundings.

\section{Release of cells from swarm colonies facilitates dissemination of $\boldsymbol{V}$. parahaemolyticus in the environment and its reattachment to new submerged surfaces}

To test if cells released from the swarm colony into the liquid environment were able to spread and reattach to new solid surfaces, we measured the ability of released cells to attach to submerged chitin flakes over time. Indeed, there was a time-course dependent increase in chitin-attached cells that correlated with the increase in released cells into the liquid surroundings (Fig. 1b). To further analyze the efficiency by which released cells are able to spread in the environment and attach to chitin, we collected released cells and transferred them to a new test tube. We then added chitin flakes and followed attachment and presence of planktonic cells over time-this allowed us to assay for attachment efficiency of released cells without the continuous contribution from the release of cells from swarm colonies. Indeed, there was a time-dependent increase in the amount of chitin-attached cells (Fig. 1c). These experiments further confirm that cells released from flooded swarm colonies have a high ability to spread in the environment and reattach to new surfaces such as chitin.

\section{Elongated swarmer cells remain surface attached and only a short-cell type is released during swarm colony flooding}

As swarm colonies primarily consist of short cells in the center and fully morphologically differentiated elongated cells in the periphery, one could expect a heterogeneous population of released cells. Thus, we analyzed the cell lengths and cell length distribution of released cells and compared them to that of cells from the center and periphery of swarm colonies and to that of planktonic cells. As expected, cells in the center were short with little variance in size while cells from the periphery were highly elongated and importantly showed a high variance in their cell length distribution (Fig. 2a-c). However, surprisingly, the cell length distribution of released cells was very homogenous and almost no long cells were detected (Fig. 2a-c). Thus, showing that long swarmer cells are not released into the liquid environment, but instead remain surface attached during flooding events. Importantly, released cells were on average also significantly different in their cell length distribution from cells found in the center of the swarm colony and they were most similar in size to planktonic cells (Fig. 2a-c). To test if incubation in our release buffer could influence the cell length during the course of the release experiment, we analyzed the cell length of cells collected from the center and periphery of swarm colonies, respectively, subsequent to their incubation in release buffer and compared these with the cell length of released cells. Again, released cells were significantly different in their average cell length and cell length variance, when compared with cells from the center or the periphery of swarm colonies after incubation in release buffer (Fig. 2b, c). Altogether, these experiments show that cells released from swarm colonies are significantly different in size from the two types of cells generally found in the center and periphery of swarm colonies, respectively, suggesting that released cells comprise a different cell type.

\section{Cells released from swarm colonies comprise a distinct cell type}

We next analyzed how cells released from swarm colonies into the liquid environment are related to the populations found within mature colonies. Particularly, we performed proteomic analysis of cells from the center $(\mathrm{C})$ and cells from the flares in the periphery $(\mathrm{P})$ of swarm colonies and compared these to that of cells released into the liquid environment (R) (Fig. 3a-c). Strikingly, the results showed that released cells have a proteomic profile distinct from both cells in the center (Fig. 3a, b) and the periphery (Fig. 3a, c) of swarm colonies. However, regardless of these significant differences, the released 
Fig. 2 Cells released from swarm colonies have a distinct morphology distinct from elongated swarmer cells. a DIC microscopy showing the morphology of planktonic cells, cells collected from the center and periphery of swarm colonies, and cells released from flooded swarm colonies. Scale bar- $-5 \mu \mathrm{m}$. b Graph showing the cell length distribution of cells from indicated environments. Black bars indicate the mean value. $P$ value was calculated using Student's $t$ test. c Bar graph showing the variance in cell length of cells from indicated environments
A
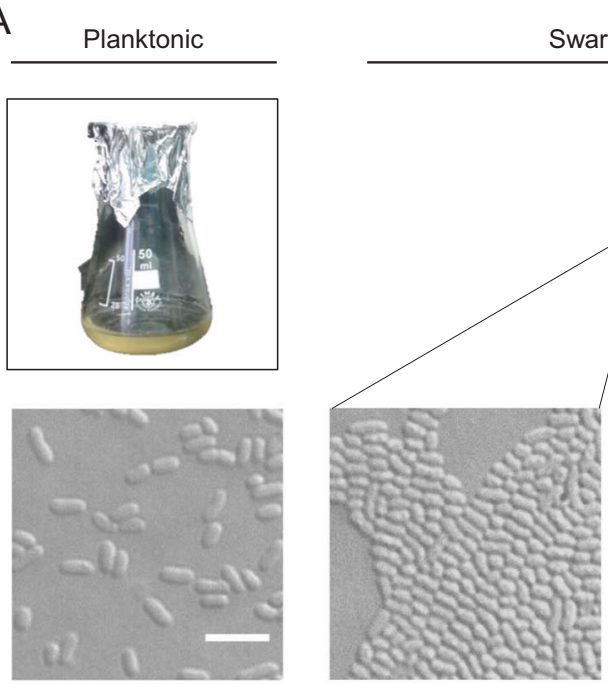

Center

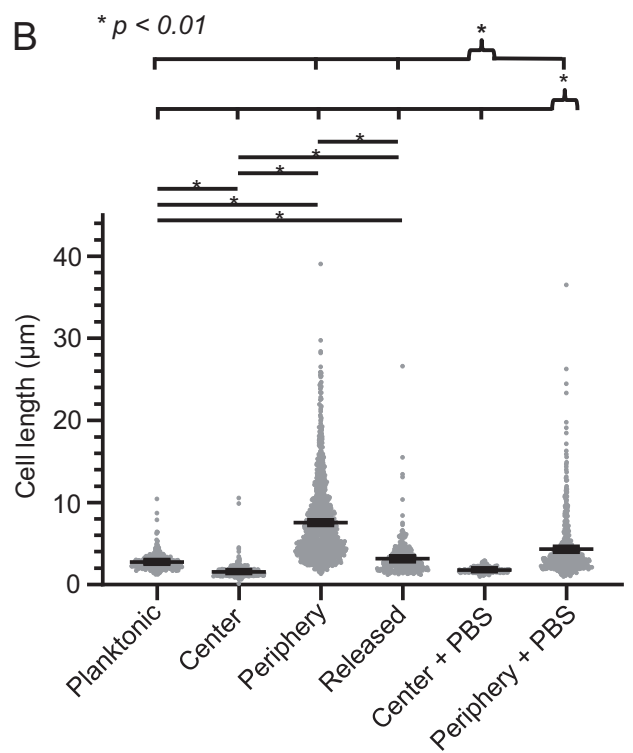

warm colony

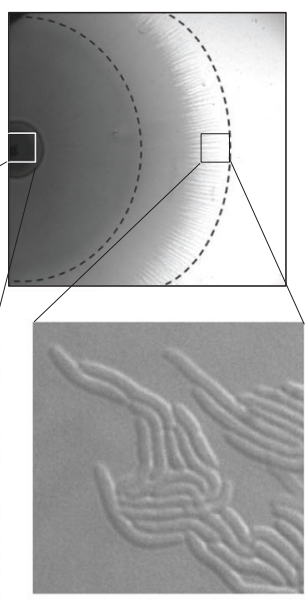

Periphery

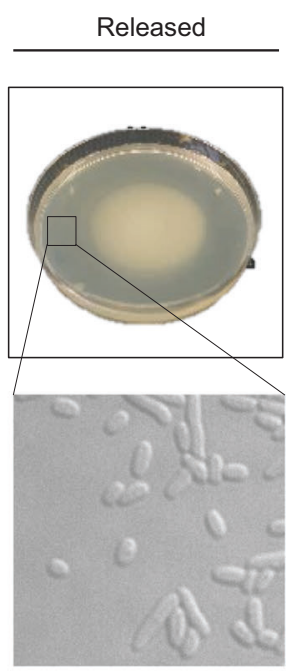

C

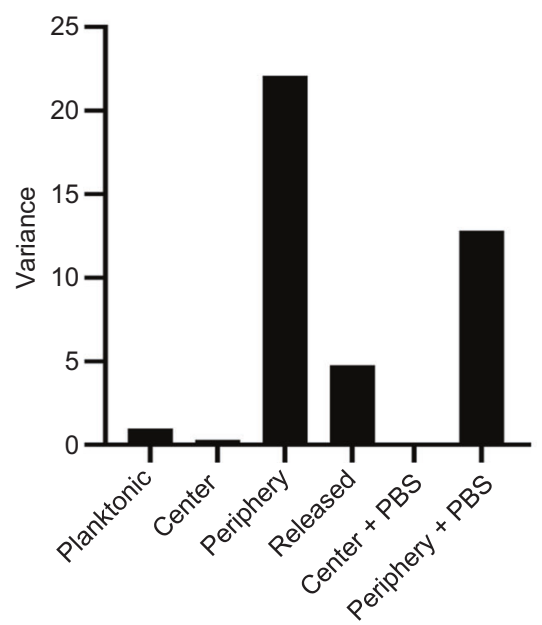

cells were more similar to cells from the center of the swarm colony when compared with those found in the periphery (Fig. 3b, c), thus suggesting that released cells likely originate from this part of mature swarm colonies. In addition, we compared released cells to planktonic cells continually cultivated in liquid medium (L), and interestingly the results showed that released cells also are highly different from planktonic cells on the proteomic level (Fig. 3a, d). Thus, showing that not only are released cells morphologically different from cells in the center and periphery of swarm flares (Fig. 2a-c), but also display a distinct proteomic profile specific to this cell type and different from both center, periphery, and planktonic cells. Interestingly, regarding the levels of proteins belonging to the type VI secretion system 1 (T6SS1), released cells contain much higher expression of these proteins when compared with planktonic cells (Fig. 3e). The fold change of the T6SS1 proteins is slightly positive when comparing to cells from the center. Furthermore, five proteins related to oxidative stress were always found to be upregulated in released cells (Fig. S1, Table S4). Altogether, this shows that cells released from the swarm colony into liquid environments are different both morphologically and proteome wise compared with cells from the center and colony periphery, respectively. Thus, indicating that released cells comprise a distinct cell type.

\section{Cells released from swarm colonies are highly swimming proficient}

Since our results show that released cells from swarm colonies are very proficient in spreading in liquid 

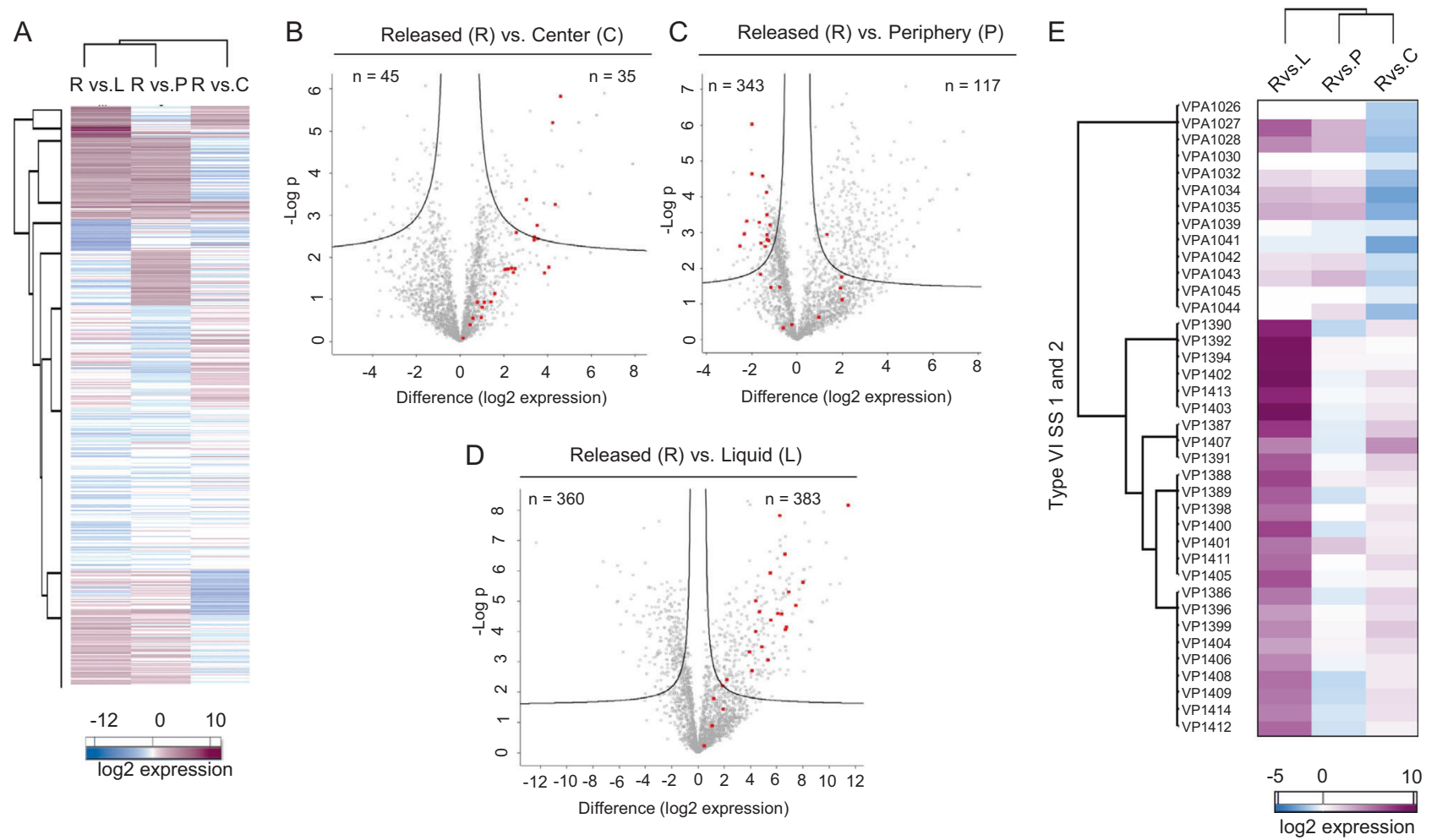

Fig. 3 Cells released from swarm colonies comprise a distinct cell type. a Clustering map depicting changes in protein intensities in three sets of comparisons. Comparison of released cells vs. liquid ( $R$ vs. L); released vs. periphery ( $\mathrm{R}$ vs. P); released vs. center ( $\mathrm{R}$ vs. L). b Volcano plot depicting changes in protein intensities in a comparison of cells from the released vs. periphery (R vs. P) of swarm colonies. c Volcano plot depicting changes in protein intensities in a comparison of cells from the released vs. center ( $R$ vs. C) of swarm colonies. d Volcano plot depicting changes in protein intensities in a comparison of cells from the released vs. liquid (R vs. L). In $\mathbf{b}-\mathbf{d}$ number " $n$ "

environments (Fig. 1b), we analyzed the swimming potential of released cells compared with that of cells obtained from the swarm colony periphery and that of planktonic cells. Particularly, we performed single-cell analysis and measured the swimming speed and displacement of individual cells as a function of cell length. The results very clearly showed that the swimming proficiency was cell length dependent. We observed both highly motile and nonmotile cells. Importantly, all highly motile cells were all shorter than $5 \mu \mathrm{m}$ in length, while cells longer than $5 \mu \mathrm{m}$ in length almost showed no swimming behavior and a significantly reduced swimming speed and displacement compared with short cells-independent of the origin of the cell within the swarm colony (Fig. 4a). Importantly, cells released from the swarm colony almost all possessed a length that correlated with a high swimming proficiency and resembled planktonic cells in respect of swimming ability-thus, showing that cells released from swarm colonies are highly swimming proficient (Fig. 4a). indicates the number of differentially expressed proteins that were significantly up- and downregulated and highlights in red indicate lateral flagellum proteins. e Clustering map depicting changes in intensities of T6SS1 and T6SS2 proteins, in three sets of comparisons. All proteins from T6SS1 start with VP\# and all proteins from T6SS2 start with VPA\#. Comparison of released vs. liquid (R vs. L); released vs. periphery ( $\mathrm{R}$ vs. P); released vs. center ( $\mathrm{R}$ vs. L). In a and e the color code indicates the difference of fold change in $\log 2$ of the intensities ratio

\section{Cells released from swarm colonies chemotax toward the chitin component $\mathrm{N}$-acetylglucosamine}

Chitin, a derivative of glucose, is a long-chain polymer of the monosaccharide $\mathrm{N}$-acetylglucosamine (GlcNAc). It is one of the primary components of the exoskeleton of crustaceans such as crabs, lobsters and shrimps, and the scales of fish. Thus, as our results show that cells released from swarm colonies are able to spread in their new liquid surroundings and subsequently reattach to chitin surfaces, we tested the ability of released cells to perform chemotaxis towards the chitin component GlcNAc and compared it with that of planktonic cells. As a negative control we included a strain deleted for the chemotaxis protein CheW $(\Delta$ cheW), which is defective in chemotactic behavior [26]. Planktonic cells showed a clear positive chemotactic response toward GlcNAc, while no chemotactic response was detected for strain $\Delta$ cheW (Fig. 4b). Thus, showing that V. parahaemolyticus RIMD 2210633 is able to sense and elicit a 

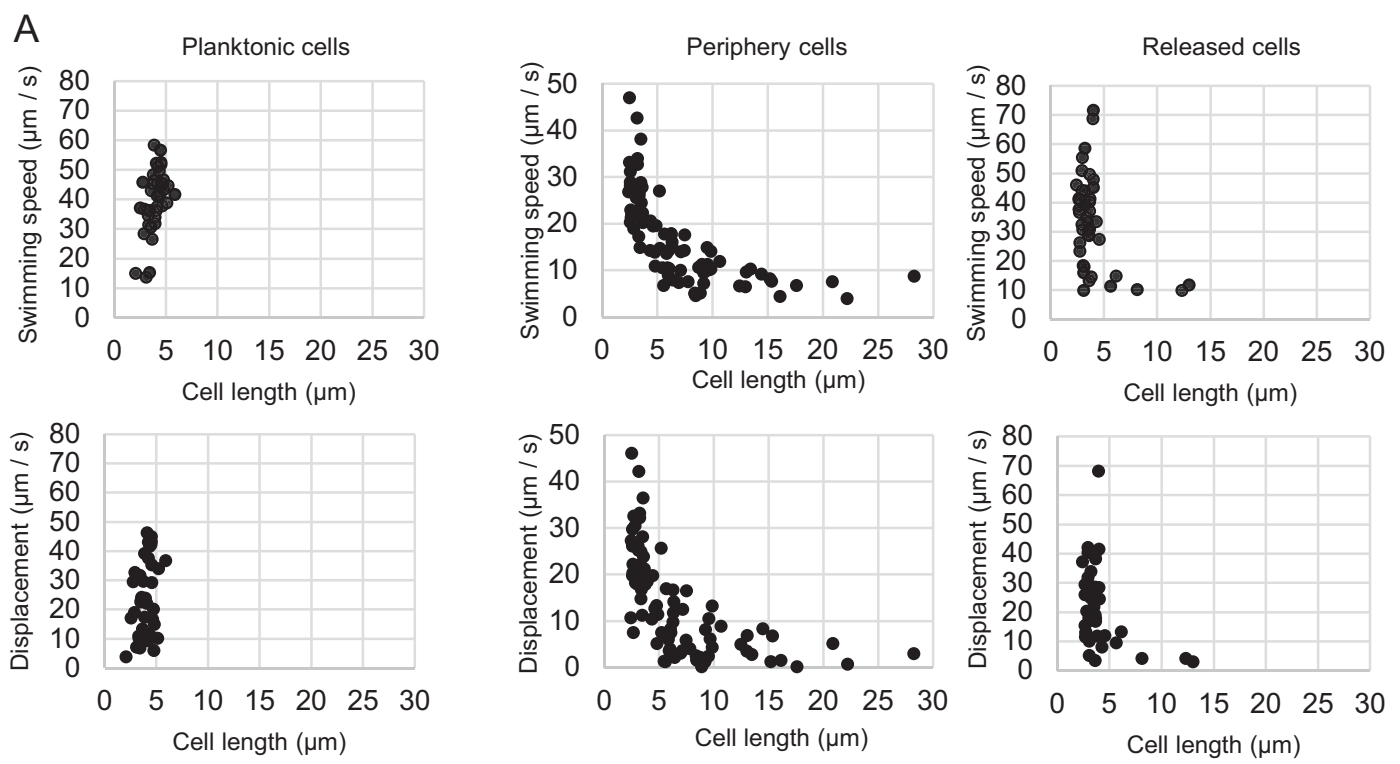

B

Chemotactic ability towards GIcNac
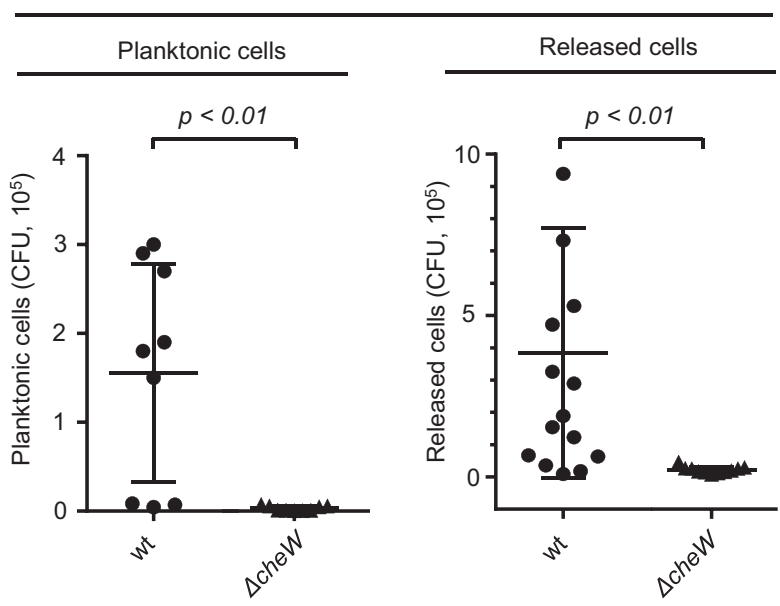

Fig. 4 Cells released from swarm colonies are highly swimming proficient and chemotax toward the chitin component $\mathrm{N}$ acetylglucosamine. a Graphs showing the swimming speed and displacement as a function of cell length. Particularly of planktonic cells, cells collected from the periphery of swarm colonies and cells released from flooded swarm colonies. b Chemotactic capillary experiment using planktonic wild-type $V$. parahaemolyticus and a
$\Delta$ cheW strain, and chemotactic capillary experiments using wildtype $V$. parahaemolyticus and $\Delta$ cheW cells released from flooded swarm colonies. Strains were assayed for chemotaxis towards Nacetylglucosamine (GlcNAc), the component of chitin polymers. Error bars indicate standard error of the mean and is based on three independent biological replicates. $P$ value was calculated using Student's $t$ test chemotactic response toward GlcNAc and thus that this chitin compound serves as a chemotactic attractant for $V$. parahaemolyticus. Importantly, similar to planktonic cells, released cells from flooded swarm colonies also displayed a positive chemotactic response toward GlcNAc (Fig. 4b). Thus, not only are cells released from swarm colonies highly swimming proficient, but they also possess the ability to perform chemotaxis toward an important nutrient source of $V$. parahaemolyticus (GlcNAc/chitin) in its environment.

\section{A distinct regional architecture of mature swarm colonies}

To further understand the function that released cells have on the spread of $V$. parahaemolyticus in the environment, we set out to analyze swarm colony development and how the swarm colony architecture depends on fluctuations in the external environment-e.g. such as flooding. To address this, we initially set out to define the swarm colony architecture and development. Particularly, we performed 
A

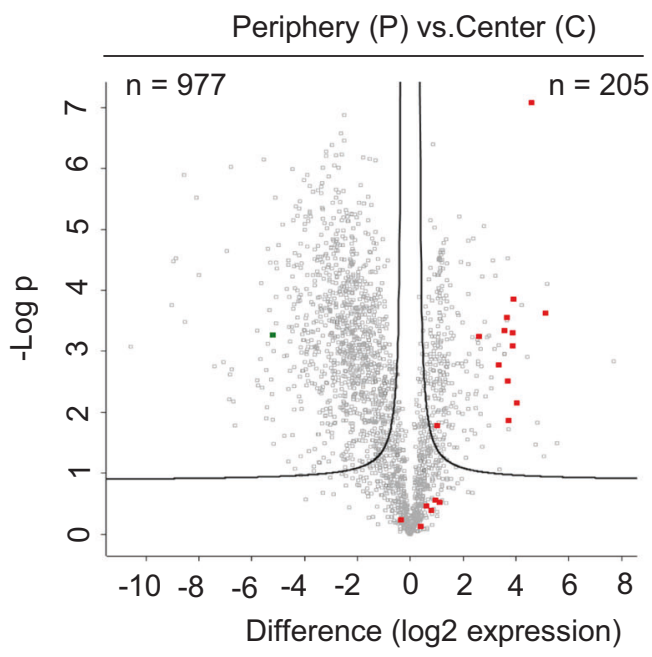

B

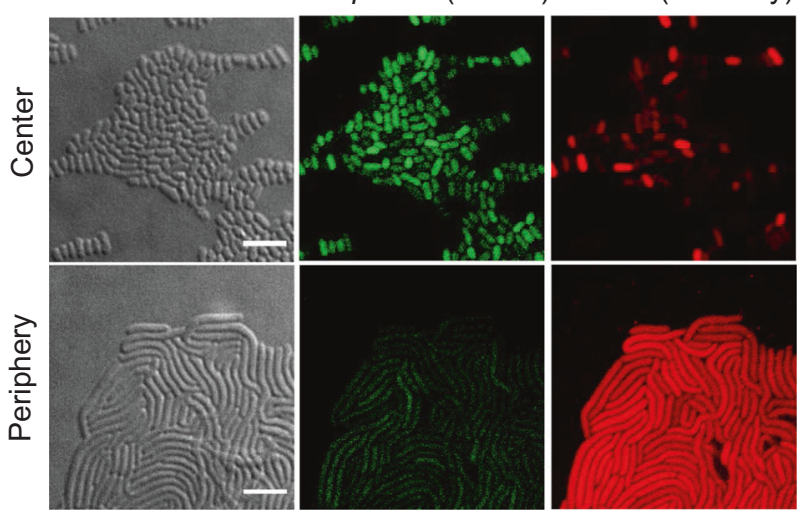

Fig. 5 A distinct zonal architecture of mature swarm colonies. a Volcano plot depicting changes in protein intensities in a comparison of cells from the periphery vs. center of swarm colonies. $X$-axis indicates the difference $(\log 2)$ of the intensities ratio. $Y$-axis indicates the $-\log$ of the $P$ value. Number " $n$ " indicates the number of differentially expressed proteins that were significantly up- and downregulated. Highlights in red indicate lateral flagellum proteins. Highlight in green indicates the protein VP1343, which is significantly downregulated in cells from the periphery. b Fluorescence and DIC microscopy of cells collected from the center and periphery of mature swarm colonies of CF36 strain. Scale bar $-5 \mu \mathrm{m}$. In CF36 the promotor of lafA was fused to mCherry in order to monitor lafA expression, and the promoter of vp1343 was fused to T7 polymerase and the promotor of $T 7$ polymerase was fused to $s f G F P$ (Pvp1343::T7 and Pt7::sfGFP) in order to monitor $v p 1343$ expression

proteomic analysis on cells collected from the center and periphery of swarm colonies (Fig. 5a). From the 2077 identified proteins, 1182 were found differentially altered, including 977 downregulated and 205 upregulated in swarmer cells from the periphery compared with cells from the center of the colony (Fig. 5a, Table S5). Interestingly, the majority of lateral flagella proteins were found to be significantly upregulated in the periphery cells, when compared with cells from the center, consistent with periphery cells also showing clear swarmer morphology (Fig. 5a in red, Table S5). These results show that not only are there morphological differences, but also significant changes in the protein expression profiles of cells depending on an individual cell's position within swarm colonies. To further analyze how distinct these regions are within colonies, we undertook a microscopy-based approach. Particularly, we transcriptionally fused the two fluorescent proteins sfGFP and mCherry, respectively, to promoters of genes encoding proteins that based on our proteomics results were upregulated in the center ( $v p 1343)$ and in the periphery (lafA) of the colony-strain CF36 $\left(\mathrm{P}_{v p 1343}:: t 7, P_{t 7}:: s f G F P\right.$, $\mathrm{P}_{\text {lafA }}:: m$ Cherry). Consistent with the proteomics results, the fluorescence microscopy showed a clear and distinct difference in expression profiles between cells from the center and the periphery (Fig. 5b). Particularly, sfGFP and mCherry expression was almost completely restricted to the center and periphery of the colony, respectively. Altogether, these results show that swarm colonies have a very structured zonal architecture and are divided into specific regions of distinct cell types - not only on the morphological level, as has been previously reported, but also on the translational level. Particularly, the results show that fully differentiated swarmer cells-i.e., they are both morphologically differentiated and have initiated the LafK dependent swarm expression program-are found in the swarm flares of the periphery of the swarm colony, while cells in the center of the colony are distinctly different and do not have the swarm program activated. Thus, mature swarm colonies have a specific architecture and are comprised of regions of distinct cell types in different morphological and proteomic states.

\section{Stage-wise architectural development of swarm colonies}

To further understand how swarm colonies develop, we followed the progression of swarm colonies over time. Particularly, we took advantage of our double labeling strain that encodes sfGFP and mCherry expression from promoters that are specifically active in the center and periphery of fully developed swarm colonies, respectively. In addition, in order to map the architectural development, swarm colony expansion was tracked using stereomicroscopy. Based on our analysis, we could define four stages, stage I-IV, of swarm colony development, which ultimately results in a fully matured swarm colony with a defined architecture that is comprised of regions of specific cell types (Fig. 6a): stage I, colony growth; stage II, differentiation initiation and swarm-flare formation; stage III, swarm-front expansion; and stage IV, swarm colony maturation and final architecture formation. Stage I was defined by a smooth colony periphery and the absence of swarm flares (Fig. 6a), all cells displayed a short-cell morphology throughout the colony and independent of their 
A
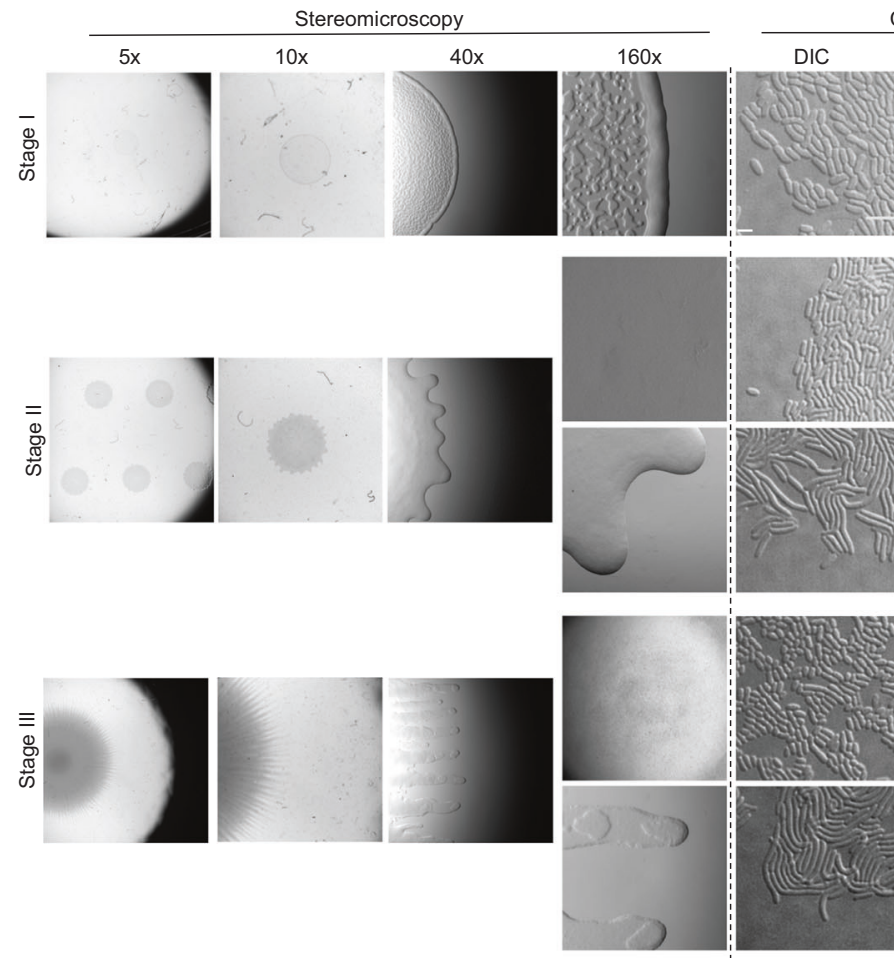

Confocal microscopy
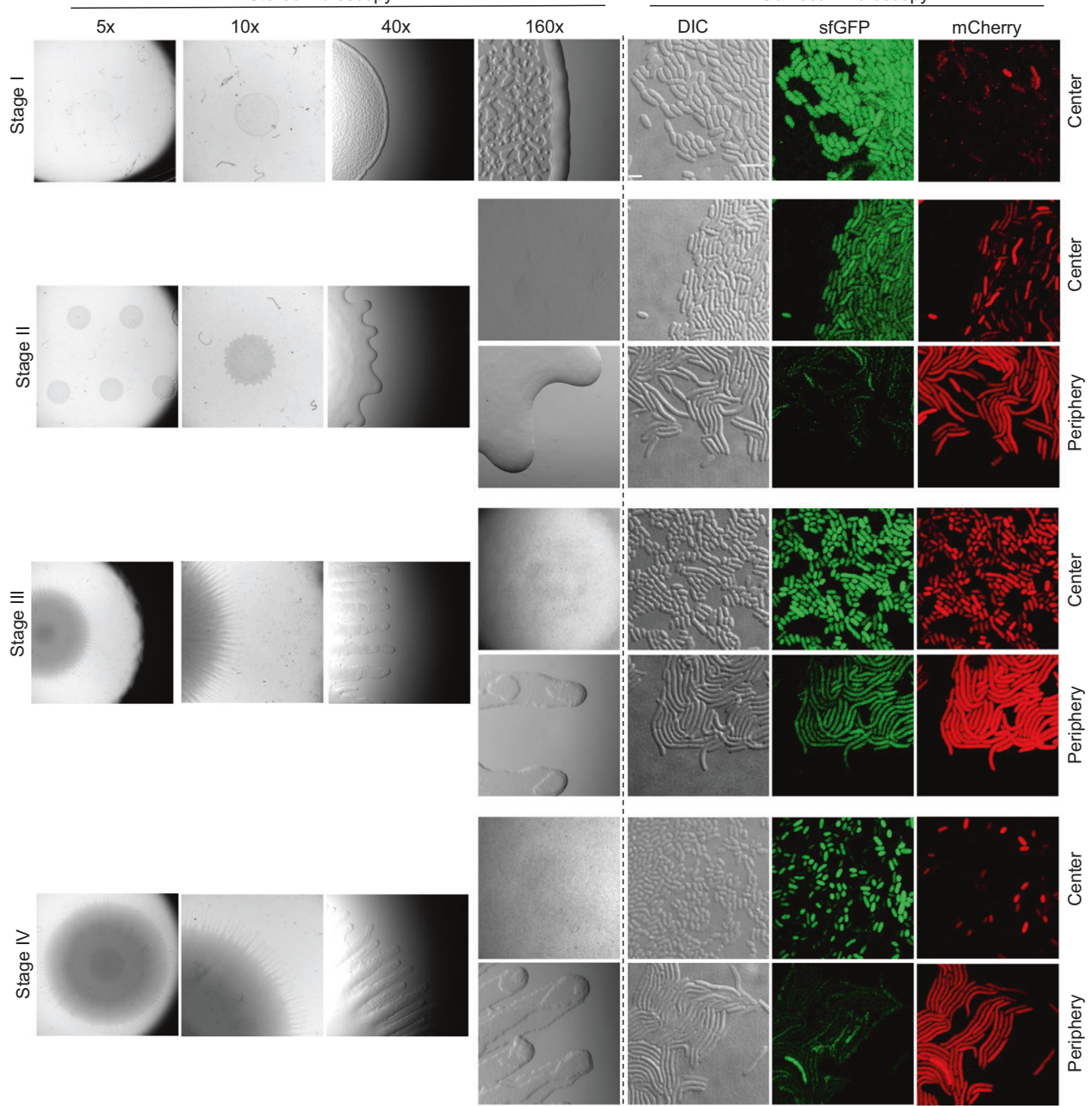

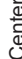

\section{$\stackrel{\Phi}{\Phi}$}

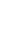

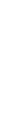

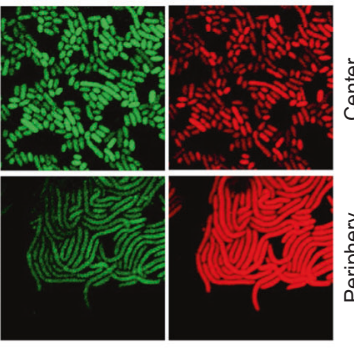

\section{.}

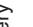
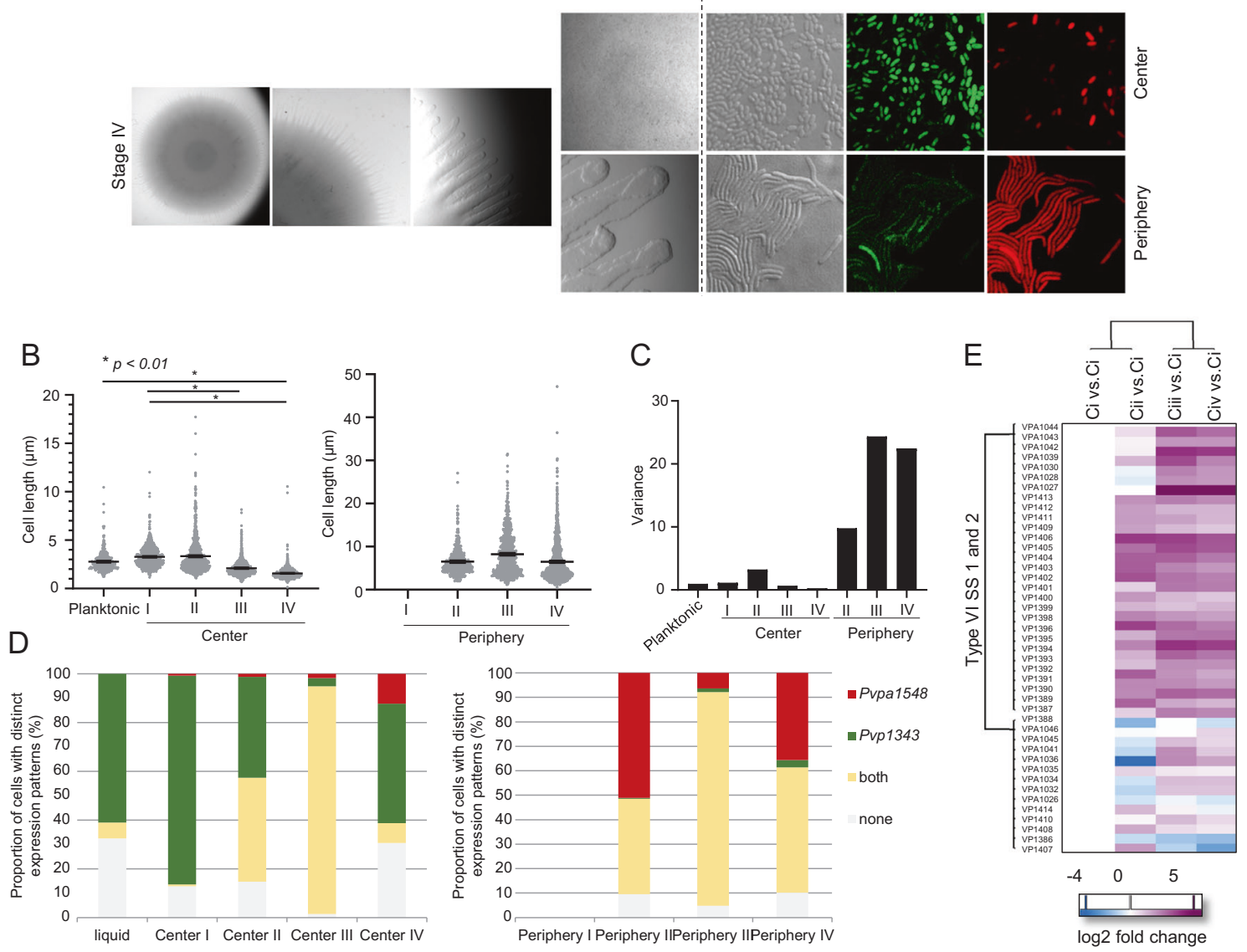

position in the center or the periphery of the colony (Fig. 6a, b). Consistently, almost no cells had initiated the swarmspecific gene program as only $1.6 \%$ were expressing

mCherry from the PlafA promoter and $\sim 90 \%$ were displaying sfGFP expression from the Pvpl343 promoter (Fig. 6a, d). Stage II was defined by differentiation initiation 
Fig. 6 Stage-wise architectural development of swarm colonies. a Stereomicroscopy imaging of swarm colony architecture during the four stages (I-IV) of swarm colony development. In addition, cells were collected from the center and periphery of swarm colonies and analyzed by DIC and fluorescence microscopy in order to evaluate cellular morphology and expression from the promoters Pvp1343 (sfGFP) and PlafA (mCherry), respectively. Scale bar- $5 \mu \mathrm{m}$. b Graph showing the cell length distribution of planktonic cells and cells from the center and periphery of swarm colonies during the four stages of swarm colony development. Black bars indicate the mean value. $P$ value was calculated using Student's $t$ test. c Bar graph showing the variance in cell length of cells from indicated environments. d Quantification of $\%$ of cells expressing mCherry and sfGFP proteins from indicated environments. Wild-type non-labeled cells from center, periphery, and liquid were imaged with the same exposure time and laser power as the CF36 strain to obtain values for auto fluorescence. The 95 percentile of the auto fluorescence intensity was calculated and this value was then subtracted from the fluorescence intensities of CF36 cells. As planktonic cells do not possess lateral flagella, the 95 percentile of the mCherry intensity from planktonic cells was calculated and this value was also subtracted from the fluorescence intensities of CF36 cells. e Clustering map depicting changes in protein intensities in three sets of comparison of proteins of T6SS1 and T6SS2. All proteins from T6SS1 start with VP\# and all proteins from T6SS2 start with VPA\#. Comparison of periphery vs. liquid (P vs. L); periphery vs. center (P vs. C)

and swarm-flare formation (Fig. 6a). In the swarm flares, cells displayed an elongated-cell morphology (average of $6.5 \mu \mathrm{m}$ in cell length) and a bigger variance in their cell sizes compared with the cells in the center of the colony that displayed an average of $3.3 \mu \mathrm{m}$ in cell length and a small variance (Fig. 6a-c). Importantly, swarmer cell differentiation was initiated throughout the colony, with $\sim 50 \%$ of cells expressing mCherry in the center of the colony and $\sim 90 \%$ of cells expressing mCherry in the developing swarm flares. sfGFP expression from the Pvpl343 promoter was detected in $\sim 85 \%$ of cells in the center and in only $\sim 40 \%$ of cells from the periphery of the colony (Fig. 6a, d). Stage III consisted of swarm-front expansion (Fig. 6a). Interestingly, cell sizes were more segregated at this stage, with cells from the center displaying a decrease in average cell length $(2.1$ $\mu \mathrm{m})$ and cells from the swarm flares displaying an increase in average cell length $(8.2 \mu \mathrm{m})$, when comparing to the previous stage II. In addition, cells in the swarm flares displayed a significantly bigger variance in their cell sizes when compared with cells from the center and cells from the periphery in stage II colonies (Fig. 6c). Swarmer cell differentiation was present throughout the colony, with $\sim 95 \%$ of cells expressing mCherry in both center and periphery regions of the colony. sfGFP expression from the Pvpl343 promoter was detected in $\sim 100 \%$ of cells in the center and $\sim 90 \%$ of cells in the expanding flares in the periphery of the colony (Fig. 6a, d). Stage IV was characterized by swarm colony maturation and final architecture formation (Fig. 6a), where cells from the center displayed the shortest cell length average of all stages $(1.6 \mu \mathrm{m})$. Cells from the flares maintained their elongated-cell morphology with an average of $6.5 \mu \mathrm{m}$ in cell length and a significantly higher variance in cell length when compared with cells in the center (Fig. 6a-c). Importantly, at this last stage there was a distinct difference in expression profiles between cells from the center and the periphery, with only $\sim 20 \%$ of cells expressing mCherry in the center of the colony and $\sim 90 \%$ expressing mCherry in the swarm flares in the periphery of the colony. Although the percentage of cells expressing sfGFP from the Pvp1343 promoter was similar between cells in the center $(\sim 60 \%)$ and in the periphery $(\sim 55 \%)$, the intensity of the sfGFP signal was significantly stronger in the cells from the center when compared with cells in the periphery (Fig. S2). Only $0.6 \%$ of cells from the periphery, compared with $22 \%$ of cells from the center, emitted a fluorescence intensity higher than 75 A.U (Fig. S2). Thus, these results show that during its development a swarm colony encompasses distinct regions of cells that display fluctuations in their expression profile, ultimately resulting in a final mature stage (stage IV) where the colony consists of two distinct regions with a clear difference between expression profiles and cellular morphology.

In addition, we performed proteomics on cells in the center of the swarm colony in the four stages of swarm colony development. From the 2885 identified proteins, the number of differentially altered proteins increased with the progression of the colony development. Particularly, 319 proteins were differentially expressed when comparing stage II with stage I (Cii vs. Ci), but this number rose to 1321 and 1418 in stages III and IV, respectively, in comparison with stage I (Ciii vs. $\mathrm{Ci}$ and $\mathrm{Civ}$ vs. $\mathrm{Ci}$ ). Moreover, 81 proteins were upregulated and 74 proteins were downregulated in cells from the center of the colony during stages II, III, and IV when compared with stage I (Table S6). Interestingly, many of the proteins whose expression continuously increased during development were proteins of the T6SS1 (Fig. 6e). As $V$. parahaemolyticus T6SS1 confers virulence against other Gram-negative bacteria [28], our results suggest that cells under swarm induction conditions seem to be activated for an antibacterial program mechanism.

\section{V. parahaemolyticus colonies act as recurrent sources of swarmer cells during fluctuations in the external environment}

Next we asked what the consequence is on swarm colony development and architecture, if the colony fluctuates between distinct environments that either stimulate or suppress swarming behavior. To this end, swarming was first induced and after reaching stage IV of development, the colony was transferred to non-swarming conditions and imaged by stereo and confocal fluorescence microscopy $5 \mathrm{~h}$ 

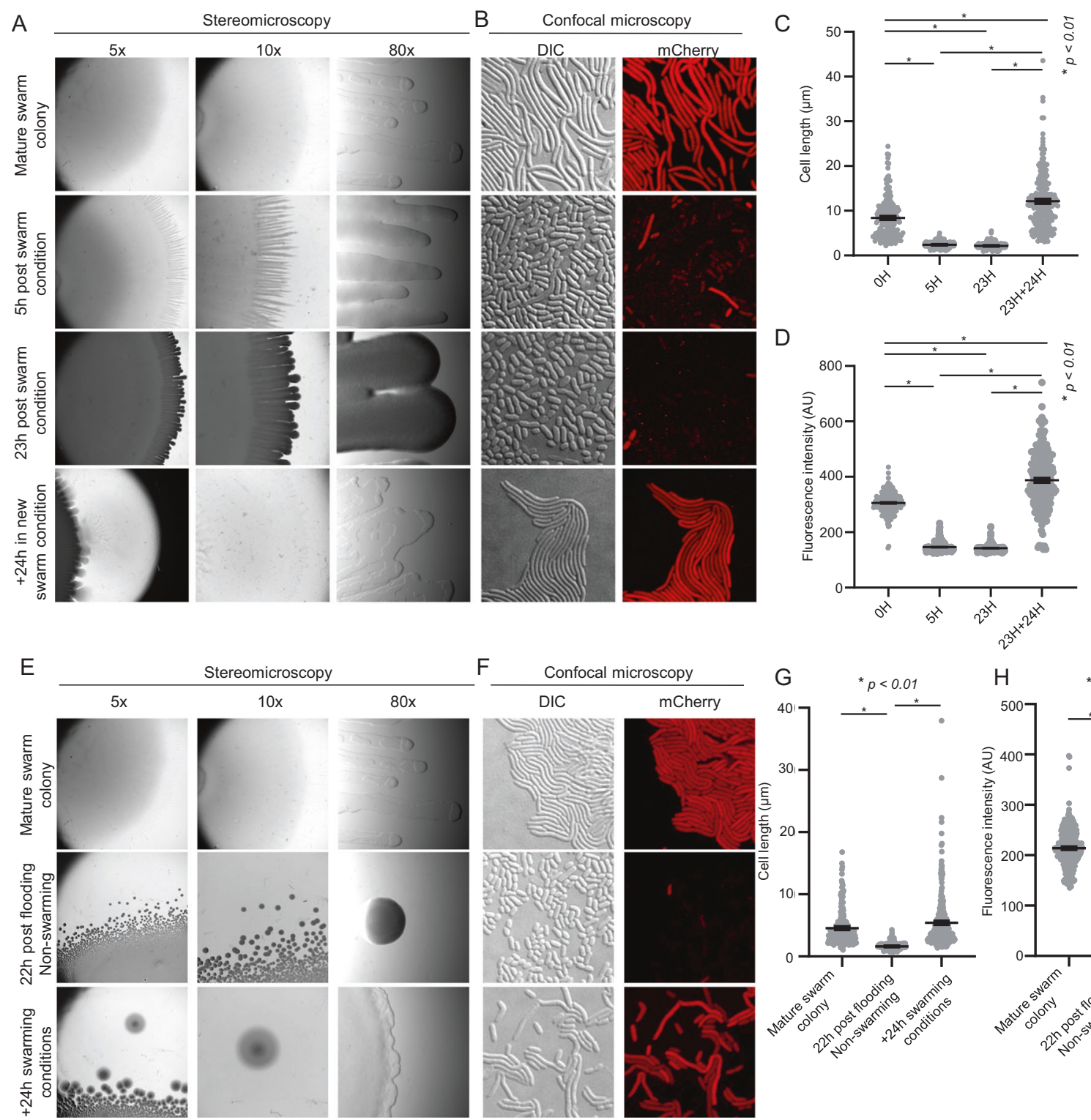

Fig. $7 \mathrm{~V}$. parahaemolyticus colonies act as recurrent sources of swarmer cells during fluctuations in the external environment. Stereomicroscopy imaging of swarm colony architecture (a) and DIC and fluorescence microscopy (b) in order to evaluate cellular morphology and expression from the swarm-specific promoter PlafA (mCherry). Scale bar $-5 \mu \mathrm{m}$. c Graph showing the fluorescence intensity (A.U) distribution of cells from the periphery of colonies imaged in b. d Graph showing the cell length distribution of cells from the periphery

and $23 \mathrm{~h}$ post transfer. Subsequently, the colony was then transferred back to swarm-inducing environments and imaged after $24 \mathrm{~h}$. The experiments showed significant changes in colony architecture and in its composition of cell types as a consequence of the environmental changes (Fig. 7a-d). Following transfer to non-swarming conditions there was a clear cessation to swarming expansion and an increase in cell density within swarm flares produced during the initial swarm colony expansion (Fig. 7a). Furthermore,
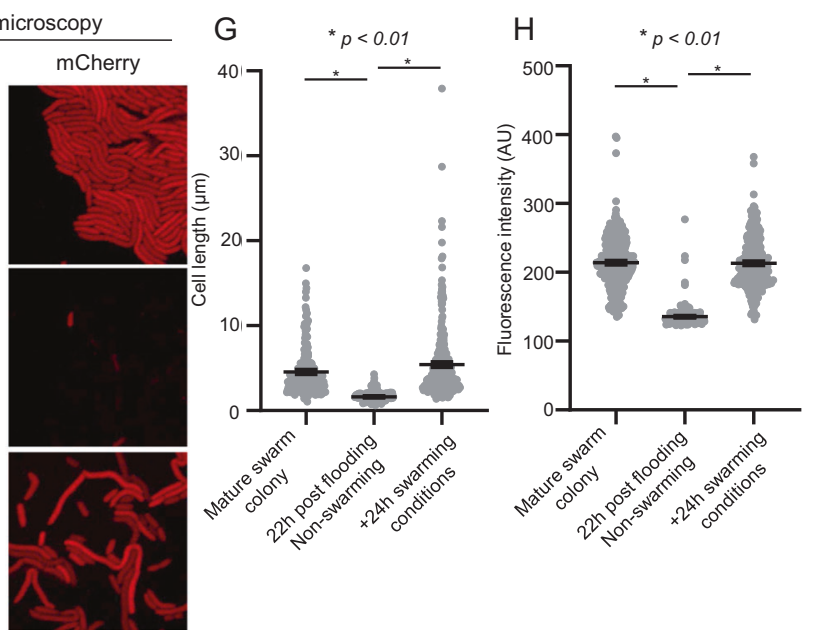

of colonies imaged in a and b. Stereomicroscopy imaging of swarm colony architecture (e) and DIC and fluorescence microscopy (f) in order to evaluate cellular morphology and expression from the swarmspecific promoter PlafA (mCherry). g Cell length distribution of cells from the periphery of colonies imaged in $\mathbf{e}$ and $\mathbf{f}$. $\mathbf{h}$ Graph showing the fluorescence intensity (A.U) distribution of cells from the periphery of colonies imaged in $\mathbf{f}$. In $\mathbf{c}, \mathbf{d}, \mathbf{g}$, and $\mathbf{h}$ black bars indicate the mean value, and $P$ value was calculated using Student's $t$-test

cells from flares experienced a change in their morphology from being highly elongated during the initial swarming and showing high expression from the PlafA promoter, to a short-cell type similar in length to that of cells from the center of swarm colonies (Fig. 7b, c) and did no longer express from the PlafA promoter (Fig. $7 \mathrm{~b}, \mathrm{~d}$ ). When the colony once again was transferred to swarm stimulating conditions new swarm flares formed and spread from the periphery of the colony (Fig. 7a) and cells from flares 
showed full morphological swarmer differentiation (Fig. 7b, c) and had initiated the swarm program (Fig. 7b, d). Interestingly, a similar fluctuation between differentiation/dedifferentiation and corresponding swarm expansion/ swarming cessation occurred when swarm colonies were subjected to flooding, and subsequent drying and transfer to swarm stimulating conditions (Fig. 7e-h). Importantly, subsequent to flooding multiple satellite colonies formed at various distances from the mother colony. When transferred to swarm-inducing conditions cells from all of these satellite colonies induced the swarm program and differentiated into swarmer cells, resulting in swarming expansion of the colony (Fig. 7e-h). Altogether, these results show that $V$. parahaemolyticus colonies act as recurrent sources of swarmer cells during fluctuations in the external environment and that the colony itself undergoes significant architectural changes with specific differentiation and dedifferentiation events within the colony flares.

\section{Discussion}

Here we show that $V$. parahaemolyticus swarm colonies undergo a specific stage-wise development that ultimately results in a highly structured architecture with regions of distinct cells that are organized into zones of different differentiation states. Importantly, we show that cells are continuously released from flooded swarm colonies into the liquid environments and thus indicating that swarm colonies function as a continuous source of cells that can be released into the environment upon colony flooding. Surprisingly, our results indicate that long swarmer cells are not released into the liquid environment but stay surface attached during flooding. Instead, released cells comprise a distinct cell type that is morphologically optimized for swimming behavior. Particularly, this distinct cell type is highly swimming proficient and allows for spreading of $V$. parahaemolyticus in the liquid environment and ultimately surface reattachment to new solid surfaces - such as chitin, thus facilitating the dissemination of $V$. parahaemolyticus in the environment-we define cells belonging to this group as a population of "adventurer" cells.

Our results indicate that formation of specific cell types within the swarm colony allows for swarming across surfaces while maintaining a subpopulation of adventurer cells that are ready to be released into liquid environments and immediately capable of exploring new surroundings. This result is in agreement with the finding that chemotaxis arrays always are positioned bipolarly in cells from swarm colonies [26]. This will in turn ensure that each daughter cell inherits an array upon cell division and thus that adventurer cells will be able to respond immediately to changes in their external milieu when released from the swarm colony into their liquid surroundings. Consistent with this, we show that released adventurer cells are chemotaxis proficient and show a positive chemotactic response toward the chitin component GlcNAc. Furthermore, we show that released cells are capable of reattaching to new surfaces such as chitin and spawn new colonies that depending on their environment also will result in the formation of swarm colonies and fast expansion of the colony over solid surfaces. V. parahaemolyticus is found free swimming, attached to underwater surfaces or associated with various species of shellfish and zooplankton [1]. This zooplankton may operate as a vehicle for dispersal of $V$. parahaemolyticus populations in the open sea, drifting along with the ocean currents, connecting distant regions and habitats, and thereby producing impacts on bacterial community demography and epidemiology. As our results show that released adventurer cells are highly swimming proficient, able to chemotaxis toward chitin compounds and ultimately attach to chitin surfaces, we speculate that the formation and release of adventurer cells from surface attached swarm colonies might also contribute to the spread of $V$. parahaemolyticus cells amongst shellfish and zooplankton and consequently further the dissemination of $V$. parahaemolyticus in the environment via this route. Indeed, studies of oysters growing in the intertidal zone found that the concentration of total and potentially pathogenic (tdh and/or trh positive) V. parahaemolyticus cells increased when oysters were exposed on the sunny mudflats by a receding tide, then decreased when the tidal waters covered the shellfish [21]. Another study revealed that $V$. parahaemolyticus is $4-8$ times higher concentrated at maximum intertidal exposure than at the beginning [22]. After reimmersion for one tidal cycle, it was shown that Vibrio levels returned to background levels [21]. The decrease of attached bacteria once the tide comes up suggests that the cells attached to the oysters are released into the liquid milieu when the tide comes in and the surface attached cells are flooded-a behavior that is consistent with the results we present in this report, where specific cells are released from swarm colonies upon swarm colony flooding. Particularly, here we show that cells attached to surfaces in swarm colonies act as reservoirs for the release of swimming proficient cells into liquid environments. Based on our results, we speculate that release of adventurer cells from surface attached swarm colonies in estuarine areas could facilitate the dissemination of $V$. parahaemolyticus when fluctuations of flooding and nonflooding occurs on surfaces, as a result of the tidal rhythm. In support of this, we show that swarm colonies undergo distinct changes in their architecture during such environmental fluctuations. Particularly, we have characterized the architecture of $V$. parahaemolyticus swarm colonies over time and shown that development and 
differentiation on the colony level is divided into four specific stages-stage I-IV: (I) colony growth, (II) differentiation initiation and swarm-flare formation, (III) swarmfront expansion, and (IV) swarm colony maturation and final architecture formation. We further show that when $V$. parahaemolyticus swarm colonies experience fluctuations in the external environment-i.e., fluctuations between swarming and swarm-suppressing conditions or colony flooding - the colony undergoes significant changes in its architecture with specific differentiation and dedifferentiation events within the colony flares. Particularly, when a switch from swarming to non-swarming conditions occurs, cells in swarm flares terminate the gene-expression program required for swarming behavior and undergoes morphological dedifferentiation. Once conditions again are swarming favorable, the swarm program is once again initiated at the colony periphery and cells undergo morphological differentiation and form new swarm flares that spread across the solid surface. Interestingly, our results also show that once swarm colonies are flooded, cells released from swarm colonies are able to spread and spawn new daughter colonies that, when the conditions are favorable, are able to initiate swarmer differentiation-hereby facilitating the colonization and distribution of $V$. parahaemolyticus over new surfaces. Thus, our results indicate that $V$. parahaemolyticus colonies act as recurrent sources of swarmer cells during fluctuations in the external environment.

The precise origin of the released cells from within the swarm colony remains an open question. However, our proteomics data show that released cells are more similar to cells from the center of the swarm colony than those found in the periphery (Fig. 3b, c) thus suggesting that released cells most likely originate from the center of the colony. Further studies are required to determine the origin and position of released cells (adventurer cells) within the swarm colony.

There are several examples of bacterial species that release cells from submerged biofilms into the surroundings and this is dependent on specific trigger signals [29-33]. One remaining question is what factors signal the release of adventurer cells from the swarm colony into the surroundings. According to our proteomic results, proteins related to oxidative stress are specifically upregulated in released cells. This suggests that bacteria residing at various regions of the swarm colony might experience different levels of oxidative tensions, which will induce the production of proteins that can restore cell redox homeostasis. The fact that these proteins were upregulated specifically in the released cells suggests that reactive oxygen species might be one of the signals involved in dispersal of cells from the swarm colony of $V$. parahaemolyticus.

The T6SS1 is recognized as an important virulence and inter-bacterial competition mechanism in several Gram- negative species [34-37]. V. parahaemolyticus T6SS1 is active under marine like conditions and it confers antibacterial virulence against other Gram-negative bacteria, including the human pathogen Vibrio cholerae [28]. In our study, levels of T6SS1 proteins were continuously increasing in the cells from the center during swarm colony development (Table S6). Our data suggest that the $V$. parahaemolyticus T6SS1 machinery is induced during swarming conditions, which could enable this bacterium to succeed in invading the solid territory by killing other colonizing bacteria. This hypothesis is further supported by the fact that once attached to chitin surfaces, the $V$. cholera T6SS1 is activated (alongside with a natural competence mechanism) and is used as an antibacterial weapon [38]. Interestingly, as the proteome of released cells is also enriched in T6SS1 proteins, when compared with planktonic cells, released cells seem to be prepared to compete with other colonizers once they reach and attach to the next surface location. A parallel comparison can be made with Streptococcus pneumoniae biofilm dispersed cells that show a pattern of virulence gene expression that will help them in their next environmental context- the host-allowing them to invade and kill human respiratory epithelial cells more effectively and to induce high pro-inflammatory cytokine responses [39].

Furthermore, the findings in this study can aid the understanding of $V$. parahaemolyticus dissemination and density on seafood, which could be of potential help in the management of $V$. parahaemolyticus. $V$. parahaemolyticus has been known to cause significant problems in the aquaculture industry with early mortality syndrome (EMS) of shrimps-an important shrimp disease in Southeast Asia [40]. In this study, we have shown that swarm colonies are an important source for release of specific adventurer cells, which are able to spread in their liquid environment and to attach to chitin surfaces, which is one of the primary components of the exoskeletons of crustaceans. Thus, the formation of swarm colonies might have a relevant impact in the aquaculture industry regarding the EMS of shrimps. Furthermore, as we show that T6SS1 is upregulated in released cells, this system could be potentially used as a marker for identification of $V$. parahaemolyticus. Furthermore, a better understanding of the importance of T6SS1 could further aid the management of Vibrios.

The fact that many bacterial species display swarming motility in the laboratory and thus possess mechanisms to override surface obstructions indicates that swarming behavior must be an important means of invading and colonizing more surface area in their natural habitats [41-45]. Vibrio alginolyticus, a close relative to V. parahaemolyticus, can also differentiate into elongated swarmer cells with multiple lateral flagella once it encounters solid surfaces [18]. These two species are among the most 
frequently encountered marine bacteria [46]. $V$. alginolyticus is an opportunistic pathogen causing ear infection and intestinal disease in humans and high-mortality vibriosis in marine animals [47, 48]. Here we describe how swarm colonies facilitate the dissemination of $V$. parahaemolyticus in the environment and its reattachment to new surfaces. We argue that swarm colonies from other pathogenic bacteria living in estuarine areas, such as $V$. alginolyticus, might also utilize a similar spreading mechanism like V. parahaemolyticus based on the release of distinct swimming proficient cells from swarm colonies-facilitating its dissemination and prevalence in the environment. Particularly, in fluctuating environments, where surfaces upon which the swarm colony is attached, experience periodic flooding.

Thus, we speculate that the work presented here could represent a general mechanism that is utilized by other swarming proficient bacteria to allow for the release of swimming proficient cells from swarm colonies and the consequential dissemination of the bacterium in the liquid environment, whilst permitting rapid surface colonization (swarming).

Acknowledgements We thank Kathrin Schirner for thoughtful comments on the manuscript and very helpful suggestions for experiments. We thank Jan Heering for construction of plasmid pJH047. This work was supported by the Max Planck Society (SR).

Author contributions CF carried out the majority of the experimental work. SR conceived the study. CF and SR designed the research and experiments and analyzed the data. CF, TG, and SR performed proteomics analysis. SR and CF wrote the manuscript.

\section{Compliance with ethical standards}

Conflict of interest The authors declare that they have no conflict of interest.

Publisher's note Springer Nature remains neutral with regard to jurisdictional claims in published maps and institutional affiliations.

\section{References}

1. McCarter L. The multiple identities of Vibrio parahaemolyticus. J Mol Microbiol Biotechnol. 1999;1:51-7.

2. Letchumanan V, Chan KG, Lee LH. Vibrio parahaemolyticus: a review on the pathogenesis, prevalence, and advance molecular identification techniques. Front Microbiol. 2014;5:705.

3. Kaneko T, Colwell RR. Adsorption of Vibrio parahaemolyticus onto chitin and copepods. Appl Environ Microbiol. 1975;29: 269-74.

4. Di DYW, Lee A, Jang J, Han D, Hur H-G. Season-specific occurrence of potentially pathogenic Vibrio spp. on the southern coast of South Korea. Appl Environ Microbiol. 2017;83:e02680-16.

5. Kaneko T, Colwell RR. Ecology of Vibrio parahaemolyticus in Chesapeake Bay. J Bacteriol. 1973;113:24-32.

6. Gamble MD, Lovell CR. Infaunal burrows are enrichment zones for Vibrio parahaemolyticus. Appl Environ Microbiol. 2011;77: 3703-14.
7. Ceccarelli D, Hasan NA, Huq A, Colwell RR. Distribution and dynamics of epidemic and pandemic Vibrio parahaemolyticus virulence factors. Front Cell Infect Microbiol. 2013;3:Article 97.

8. McCarter L, Silverman M. Surface-induced swarmer cell differentiation of Vibrio parahaemolyticus. Mol Microbiol. 1990; 4:1057-62.

9. McCarter LL. Dual flagellar systems enable motility under different circumstances. J Mol Microbiol Biotechnol. 2004;7: $18-29$.

10. McCarter LL. Bacterial acrobatics on a surface: swirling packs, collisions, and reversals during swarming. J Bacteriol. 2010;192: 3246-8.

11. Gode-Potratz CJ, Kustusch RJ, Breheny PJ, Weiss DS, McCarter LL. Surface sensing in Vibrio parahaemolyticus triggers a programme of gene expression that promotes colonization and virulence. Mol Microbiol. 2011;79:240-63.

12. Makino K, Oshima K, Kurokawa K, Yokoyama K. Genome sequence of Vibrio parahaemolyticus: a pathogenic mechanism distinct from that of $V$. cholerae. Lancet. 2003;361:743-9.

13. Belas MR, Colwell RR. Scanning electron microscope observation of the swarming phenomenon of Vibrio parahaemolyticus. J Bacteriol. 1982;150:956-9.

14. Roth D, Finkelshtein A, Ingham C, Helman Y, Sirota-Madi A, Brodsky L, et al. Identification and characterization of a highly motile and antibiotic refractory subpopulation involved in the expansion of swarming colonies of Paenibacillus vortex. Environ Microbiol. 2013;15:2532-44.

15. Muraleedharan S, Freitas C, Mann P, Glatter T, Ringgaard S. A cell length-dependent transition in MinD-dynamics promotes a switch in division-site placement and preservation of proliferating elongated Vibrio parahaemolyticus swarmer cells. Mol Microbiol. 2018;109:365-84.

16. Be'er A, Strain SK, Hernández RA, Ben-Jacob E, Florin E-L. Periodic reversals in Paenibacillus dendritiformis swarming. $\mathrm{J}$ Bacteriol. 2013;195:2709-17.

17. Harshey RM, Partridge JD. Shelter in a swarm. J Mol Biol. 2015;427:3683-94.

18. Böttcher T, Elliott HL, Clardy J. Dynamics of snake-like swarming behavior of Vibrio alginolyticus. Biophys J. 2016;110:981-92.

19. Rauprich O, Matsushita M, Weijer CJ, F Siegert, Esipov SE, Shapiro JA. Periodic phenomena in Proteus mirabilis swarm colony development. J Bacteriol. 1996;178:6525-38.

20. Little K, Austerman J, Zheng J, Gibbs KA. Cell shape and population migration are distinct steps of Proteus mirabilis swarming that are decoupled on high-percentage agar. J Bacteriol. 2019;201:e00726-18.

21. Jones JL, Kinsey TP, Johnson LW, Porso R, Friedman B, Curtis M, et al. Effects of intertidal harvest practices on levels of Vibrio parahaemolyticus and Vibrio vulnificus bacteria in oysters. Appl Environ Microbiol. 2016;82:4517-22.

22. Nordstrom JL, Kaysner CA, Blackstone GM, Vickery MCL, Bowers JC, DePaola A. Effect of intertidal exposure on Vibrio parahaemolyticus levels in Pacific Northwest Oysters. J Food Prot. 2016;67:2178-82.

23. Miller VL, Mekalanos JJ. A novel suicide vector and its use in construction of insertion mutations: osmoregulation of outer membrane proteins and virulence determinants in Vibrio cholerae requires toxR. J Bacteriol. 1988;170:2575-83.

24. Donnenberg MS, Kaper JB. Construction of an Eae deletion mutant of enteropathogenic Escherichia coli by using a positiveselection suicide vector. Infect Immun. 1991;59:4310-7.

25. Heering J, Alvarado A, Ringgaard S. Induction of cellular differentiation and single cell imaging of Vibrio parahaemolyticus swimmer and swarmer cells. J Vis Exp. 2017;123:e55842. 
26. Heering J, Ringgaard S. Differential localization of chemotactic signaling arrays during the lifecycle of Vibrio parahaemolyticus. Front Microbiol. 2016;7:1767.

27. Ringgaard S, Hubbard T, Mandlik A, Davis BM, Waldor MK. RpoS and quorum sensing control expression and polar localization of Vibrio cholerae chemotaxis cluster III proteins in vitro and in vivo. Mol Microbiol. 2015;97:660-75.

28. Salomon D, Gonzalez H, Updegraff BL, Orth K. Vibrio parahaemolyticus type VI secretion system 1 is activated in marine conditions to target bacteria, and is differentially regulated from System 2. PLoS ONE. 2013;8:e61086.

29. Barraud N, Hassett DJ, Hwang SH, Rice SA, Kjelleberg S, Webb JS. Involvement of nitric oxide in biofilm dispersal of Pseudomonas aeruginosa. J Bacteriol. 2006;188:7344-53.

30. Webb JS, Thompson LS, James S, Charlton T, Tolker-Nielsen T, Koch B, et al. Cell death in Pseudomonas aeruginosa biofilm development. J Bacteriol. 2003;185:4585-92.

31. Morgan R, Kohn S, Hwang SH, Hassett DJ, Sauer K. BdlA, a chemotaxis regulator essential for biofilm dispersion in Pseudomonas aeruginosa. J Bacteriol. 2006;188:7335-43.

32. Thormann KM, Saville RM, Shukla S, Spormann AM. Induction of rapid detachment in Shewanella oneidensis MR-1 biofilms. J Bacteriol. 2005;187:1014-21.

33. Singh PK, Bartalomej S, Hartmann R, Jeckel H, Vidakovic L, Nadell CD, et al. Vibrio cholerae combines individual and collective sensing to trigger biofilm dispersal. Curr Biol. 2017;27:3359. e7

34. Miyata ST, Kitaoka M, Brooks TM, McAuley SB, Pukatzki S. Vibrio cholerae requires the type VI secretion system virulence factor vasx to kill Dictyostelium discoideum. Infect Immun. 2011;79:2941-9.

35. Schwarz S, West TE, Boyer F, Chiang WC, Carl MA, Hood RD, et al. Burkholderia type vi secretion systems have distinct roles in eukaryotic and bacterial cell interactions. PLoS Pathog. 2010;6: e100168.

36. Hood RD, Singh P, Hsu FS, Güvener T, Carl MA, Trinidad RRS, et al. A type VI secretion system of Pseudomonas aeruginosa targets a toxin to bacteria. Cell Host Microbe. 2010;7:25-37.
37. MacIntyre DL, Miyata ST, Kitaoka M, Pukatzki S. The Vibrio cholerae type VI secretion system displays antimicrobial properties. Proc Natl Acad Sci USA. 2010;107:19520-4.

38. Borgeaud S, Metzger LC, Scrinari T, Blokesch M. The type VI secretion system of Vibrio cholerae fosters horizontal gene transfer. Science. 2015;347:63-8.

39. Marks LR, Davidson BA, Knight PR, Hakansson AP. Interkingdom signaling induces Streptococcus pneumoniae biofilm dispersion and transition from asymptomatic colonization to disease. MBio. 2013;4:e00438-13.

40. Tran L, Nunan L, Redman RM, Mohney LL, Pantoja CR, Fitzsimmons K, et al. Determination of the infectious nature of the agent of acute hepatopancreatic necrosis syndrome affecting penaeid shrimp. Dis Aquat Organ. 2013;105:45-55.

41. Alberti L, Harshey RM. Differentiation of Serratia marcescens 274 into swimmer and swarmer cells. J Bacteriol. 1990;172:4322-8.

42. Kirov SM, Tassell BC, Semmler ABT, Donovan LAO, Rabaan AA, Shaw JG. Lateral flagella and swarming motility in Aeromonas species. J Bacteriol. 2002;184:547-55.

43. Harshey RM. Bees aren't the only ones: swarming in Gramnegative bacteria. Mol Microbiol. 1994;13:389-94.

44. Rather PN. Swarmer cell differentiation in Proteus mirabilis. Environ Microbiol. 2005;7:1065-73.

45. Sar N, McCarter L, Simon M, Silverman M. Chemotactic control of the two flagellar systems of Vibrio parahaemolyticus. J Bacteriol. 1990;172:334-41.

46. Böer SI, Heinemeyer E-A, Luden K, Erler R, Gerdts G, Janssen F, et al. Temporal and spatial distribution patterns of potentially pathogenic Vibrio spp. at recreational beaches of the German North Sea. Micro Ecol. 2013;65:1052-67.

47. Hornstrup MK, Gahrn-Hansen B. Extraintestinal infections caused by Vibrio parahamolyticus and Vibrio alginolyticus in a danish county, 1987-1992. Scand J Infect Dis. 1993;25: 735-40.

48. Sabir M, Ennaji Moulay M, Cohen N. Vibrio alginolyticus: an emerging pathogen of foodborne diseases. Int J Sci Technol. 2013;2:302-9. 